\title{
Contribution of oenocytes and pheromones to courtship behaviour in Drosophila
}

\author{
Claude Wicker-Thomas*1, Ilhem Guenachi ${ }^{1}$ and Youssouf F Keita ${ }^{2}$
}

\author{
Address: ${ }^{1}$ CNRS, UPR9034, LEGS and University Paris Sud, 91198 Gif sur Yvette Cedex, France and ${ }^{2}$ NAMC, UMR 9620, University Paris Sud, \\ 91405 Orsay Cedex, France \\ Email: Claude Wicker-Thomas* - Wicker@legs.cnrs-gif.fr; Ilhem Guenachi - ihcaneug@yahoo.fr; Youssouf F Keita - founefaya@yahoo.fr \\ * Corresponding author
}

Published: II August 2009

BMC Biochemistry 2009, 10:21 doi:10.1186/|47|-209|-10-21
Received: 9 April 2009

Accepted: II August 2009

This article is available from: http://www.biomedcentral.com/I47I-209I//0/2I

(C) 2009 Wicker-Thomas et al; licensee BioMed Central Ltd.

This is an Open Access article distributed under the terms of the Creative Commons Attribution License (http://creativecommons.org/licenses/by/2.0), which permits unrestricted use, distribution, and reproduction in any medium, provided the original work is properly cited.

\begin{abstract}
Background: In Drosophila, cuticular sex pheromones are long-chain unsaturated hydrocarbons synthesized from fatty acid precursors in epidermal cells called oenocytes. The species $D$. melanogaster shows sex pheromone dimorphism, with high levels of monoenes in males, and of dienes in females. Some biosynthesis enzymes are expressed both in fat body and oenocytes, rendering it difficult to estimate the exact role of oenocytes and of the transport of fatty acids from fat body to oenocytes in pheromone elaboration. To address this question, we RNAi silenced two main genes of the biosynthesis pathway, desat I and desatF, in the oenocytes of $D$. melanogaster, without modifying their fat body expression.

Results: Inactivation of desat I in oenocytes resulted in a $96 \%$ and $78 \%$ decrease in unsaturated hydrocarbons in males and females, respectively. Female pheromones (dienes) showed a decrease of $90 \%$. Inactivation of desatF, which is female-specific and responsible for diene formation, resulted in a dramatic loss of pheromones $(-98 \%)$ paralleled with a two-fold increase in monoenes. Courtship parameters (especially courtship latency) from wild-type males were more affected by desat I knocked-down females (courtship latency increased by four fold) than by desatF knockeddown ones $(+65 \%$ of courtship latency).

The number of transcripts in oenocytes was estimated at 0.32 and 0.49 attomole/ $\mu \mathrm{g}$ for desat $I$ in males and females, respectively, about half of the total transcripts in a fly. There were only 0.06 attomole/ $\mu \mathrm{g}$ desat $F$ transcripts in females, all located in the oenocytes.

Conclusion: Knock-down results for desat / suggest that there must be very little transport of unsaturated precursors from fat body to the oenocytes, so pheromone synthesis occurs almost entirely through the action of biosynthesis enzymes within the oenocytes. Courtship experiments allow us to discuss the behavioral role of diene pheromones, which, under special conditions, could be replaced by monoenes in $D$. melanogaster. A possible explanation is given of how pheromones could have evolved in species such as $D$. simulans, which only synthesize monoenes.
\end{abstract}




\section{Background}

In insects, sex pheromones, together with visual and acoustic clues, play a large role in the courtship behaviour preceding mating [1-4]. In Drosophila, pheromones are long-chain hydrocarbons which appear to be synthesized in large epidermal cells called oenocytes and deposited on the cuticle [5]. They have been widely studied and their biosynthesis is partly known: fatty acid precursors are desaturated by acyl-CoA-desaturases and elongated to give very long chain fatty acids, which are eventually decarboxylated to give hydrocarbons [6,7]. Among the Drosophila melanogaster hydrocarbons, only unsaturated ones have been shown to have a behavioral role: principally monoenes, unsaturated in position 7 in males and dienes unsaturated in positions 7 and 11 in females. The main pheromones are 7-tricosene (7-T; 23:1) and 7-pentacosene $(7-\mathrm{P} ; 25: 1)$ in males, and 7,11-heptacosadiene (7,11-HD; 27:2) and 7,11-nonacosadiene (7,11-ND; 29:2) in females. Some other hydrocarbons with one unsaturation, 7-pentacosene and 7-heptacosene (7-H; 27:1), can also elicit - at a lower level - courtship behavior $[1,8]$.

In the biosynthesis of pheromones, two desaturases can perform unsaturations: Desat 1 enzyme catalyzes the conversion of palmitic and stearic acids into palmitoleic and oleic acids, which serve as substrates for the synthesis of various lipids and also of 7 -unsaturated hydrocarbons $[9,10]$. desat 1 gene is expressed in both males and females in a number of tissues, including fat body, which is the main site of production of fatty acids, and oenocytes, which is the site of production of hydrocarbons and is involved in hydrocarbon metabolism [11]. Female fly mutants for desat 1 have fewer pheromones and are less attractive to wild-type males, leading to increased courtship latency [12-14]. In desat1 mutants, lipid metabolism is also severely impaired, and characterized by a dramatic decrease in both saturated and unsaturated fatty acid production [14]. The second enzyme is DesatF, which introduces a second double bond in the fatty acid precursors. The gene, only expressed in females, seems specifically expressed in oenocytes; a line knocked-down for desatF has been generated in the laboratory. The expression of desatF RNAi in fat body alone had no effect; the same expression induced in both oenocytes and fat body led to a large decrease in female pheromone production and also to less courtship from wild-type males [15].

As desat1 pleiotropic effects could be due to its expression in different tissues, we wanted to dissociate the effects of desat 1 in oenocytes from those in fat body. We wondered whether oenocytes could - alone - account for the synthesis of unsaturated hydrocarbons. Therefore, we used one GAL4 driver targeting expression in the oenocytes without affecting the fat body. Overexpression of desat 1 in oenocytes resulted in a small increase in unsaturated hydrocar- bons. With this same driver, flies knocked-down for desat1 showed a dramatic loss of all the unsaturated hydrocarbons. We quantified desat1 transcripts in the oenocytes by the comparison of the numbers of transcripts in flies expressing or knocked-down for desat1 in oenocytes. We also studied male courtship behavior toward the desat1 knocked-down females. We compared these effects with the effects of desatF RNAi expressed in the oenocytes. desatF knock-down in females led to a large increase in monoenes at the expense of dienes, which were almost completely eliminated. We studied the effect of these desatF knocked-down females on wild-male courtship behavior and quantified the number of desatF transcripts. Results show that wild-type male behavior toward desat1 and desatF RNAi females was very different. The role of unsaturated hydrocarbons on male courtship behavior is discussed.

\section{Results \\ Effect of desat I overexpression on hydrocarbons}

Hydrocarbons from flies overexpressing desat 1 under the control of 1407-GAL4 resulted in a small but significant increase in unsaturated hydrocarbons (+ 10 to $16 \%$ ), at the expense of saturated hydrocarbons ( -15 to $46 \%)$. In females, total diene and pheromone (HD+ND) levels were particularly enhanced (+ 23 to $28 \%$ ); in males, the pheromone level $(7-T+7-P)$ was increased from 8 to $12 \%$, depending on the line (Figure 1).

\section{Effect of desat I knock-down on hydrocarbons (Figure 2)}

When 1407-GAL4 driver was used to target desat1RNAi, no lethality occurred and flies seemed unaffected in their behavior (locomotor activity). However, their hydrocarbon profiles were dramatically affected, with an almost complete disappearance of unsaturated hydrocarbons ($96 \%$ and $-78 \%$ in males and females, respectively). The level of saturated linear hydrocarbons was multiplied by 4 in both sexes. In females, total dienes (and pheromones) were decreased by $90 \%$, monoenes by $40 \%$.

\section{Effect of desatF knock-down on hydrocarbons (Figure 3)} The RNAi silencing of desatF expression in females under 1407-GAL4 driver resulted in an increase $(+27 \%)$ in linear saturated hydrocarbons; the amount of dienes and pheromones was decreased by $98 \%$, that of monoenes was doubled.

\section{Courtship behavior}

The percentages of wild-type males performing courtship toward the two types of females (control and desat1RNAi) were similar, but courtship latency was 4 times higher when the female was desat1RNAi (Figure 4). Males attempting copulation and males succeeding in copulation were fewer (-45\% and $-70 \%$, respectively) and times required for these behaviours were increased $(+112 \%$ and $+28 \%$, respectively). The total copulation attempts were 
Males
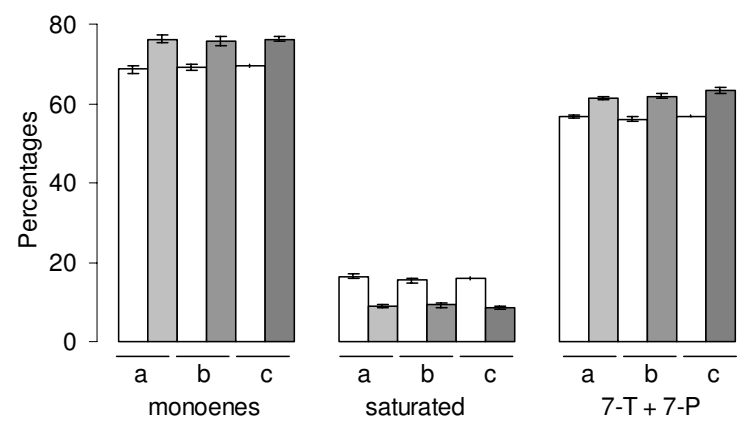

Females

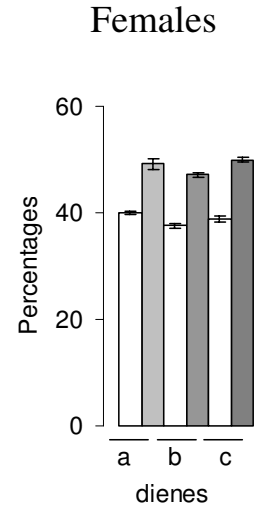

$\square$ Controls (lines a, b, c)

UAS-desat1 (line a)

UAS-desat1 (line b)

UAS-desat 1 (line c)
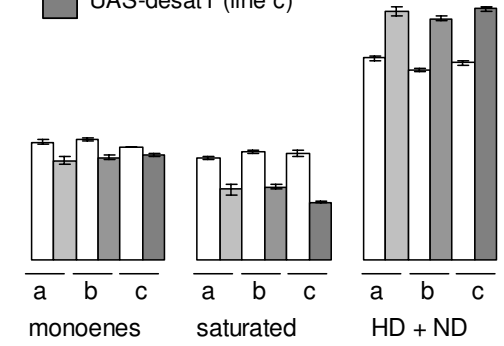

\section{Figure I}

Overexpression of desat $l$ in oenocytes results in an increase of unsaturated hydrocarbons (monoenes and dienes) and a decrease in saturated ones. Mean hydrocarbons ( \pm SEM) of 4-day-old male and female flies of wild-type (white) or expressing desat I (grey) under 1407GAL4 driver. Three different UAS-desat l lines (a, b, c) were used. $\mathrm{n}=10$ for all tests. All the hydrocarbon differences between control (I407-GAL4/+) and desat I overexpressing lines (I407-GAL4/UAS-desat I) were significantly different $(P$ $<0.05$, Student's t-test).

also decreased $(-54 \%)$ when males were in the presence of desat1RNAi females.

The courtship parameters were less affected when males were put with desatFRNAi females: there were only $12 \%$ fewer copulation attempts and 15\% less copulation, respectively. Courtship, the first copulation attempt and copulation latencies were increased by $65 \%, 115 \%$ and $55 \%$, respectively.

\section{Quantification of transcripts}

The number of desat 1 transcripts was 1.6 times higher in females than in males (Table 1). After RNAi expression under 1407-GAL4, the number of transcripts was halved in both sexes. The number of desat 1 transcripts in GAL4expressing cells was therefore estimated at 0.32 and 0.49

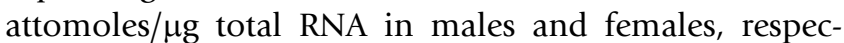
tively.

The desatF transcripts could be detected only in 1407 GAL4/+ females, and their number was evaluated at 0.06 \pm 0.01 attomoles/ $\mu$ g total RNA in 1407-GAL4/+ females.

\section{Discussion}

Results show that knock-down of desaturase genes in oenocytes is sufficient to suppress or at least largely inhibit pheromone biosynthesis. The effect is particularly clear in males, where desat 1 knock-down under 1407-GAL4 led to a $96 \%$ decrease in monoenic hydrocarbons. In females, the decrease is less dramatic (78\%). The biosynthesis of the saturated hydrocarbons was enhanced, as a consequence of the lack of desaturation of fatty acid precursors. Decreased levels of unsaturated hydrocarbons and increased levels of saturated ones have previously been described in P-desat1 mutant and resulting excision lines $[12,14]$. However, the effects on hydrocarbons were relatively smaller, ranging from a 0 to about a $66 \%$ decrease in unsaturated hydrocarbons, depending on the lines. The fact that we never obtained an almost complete loss of hydrocarbons with desat 1 mutants in former studies may be due to the pleiotropic nature of the mutation, which affects both fat body and hydrocarbons and also to the hypomorphic state of the mutant desat 1 alleles [13]. The strongest mutant phenotypes (with a three-fold decrease in male monoenes) were leaner and poorly viable (many of the mutant flies died at the larval stage), with a two-fold loss of fat in the adults [14].

By contrast, desat1 knocked-down flies under 1407-GAL4 driver were perfectly viable and showed no difference from the control ones (apart from their hydrocarbon profile). The present results obtained with desat1 RNAi expressed mainly in the oenocytes imply that most of the biosynthesis of pheromones must be made de novo in the oenocytes. A transport of fatty acids and hydrocarbons by a transporter, the lipophorin, has been described, and radio-labelled fatty acid applied topically can serve as precursors for hydrocarbon synthesis [16]. Lipophorin is essential for selective lipid transport and energy production [17]. It also delivers hydrocarbons and sex pheromones from the oenocytes to the cuticular surface and could also play an important role in delivering hydrocarbons to specific tissues such as ovaries, the digestive tract and fat body [18-20]. The transport of fatty acids to the oenocytes does occur but seems relatively low: it has been estimated at about $2-4 \%$, by comparing radioactivity found in crude extracts 3 or 6 days after topical application to that found in hydrocarbons [21] and may account for the low levels 
A

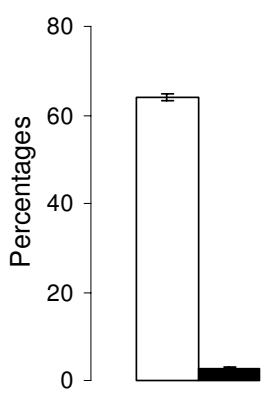

monoenes
Males

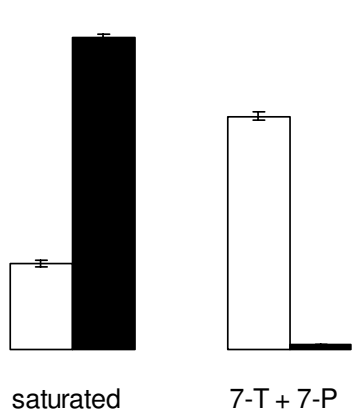

$\mathrm{B}$

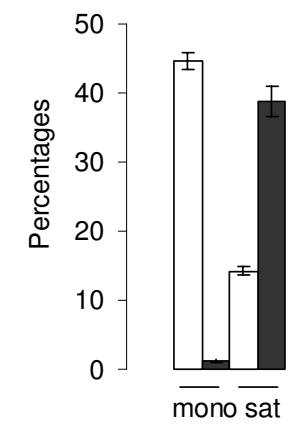

chain length

C23

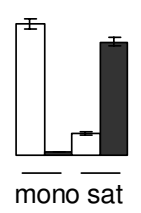

C25
C27

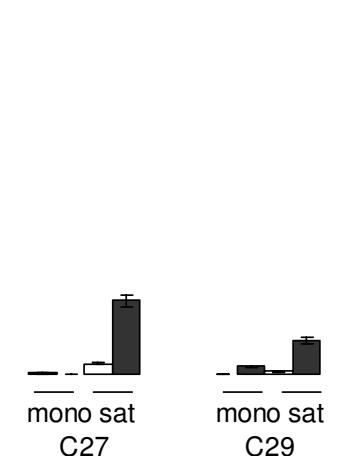

C29

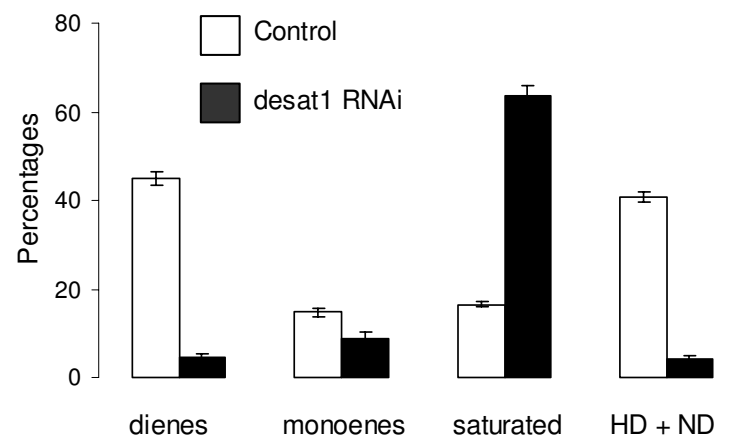

Females

\section{Figure 2}

RNAi knock-down of desat $I$ in oenocytes leads to a dramatic decrease in unsaturated hydrocarbons counterbalanced by an increase in saturated hydrocarbons. A. Percentages of different classes of hydrocarbons (dienes, monoenes, saturated, pheromones) in control (white) and RNAi (black) flies. B. Percentages of the specific hydrocarbons according to their class and their length. All the hydrocarbon differences between control (I407-GAL4/+) and desat/ knockeddown lines (I407-GAL4/UAS-desatI RNAi) were significantly different $(P<0.00$ I, Student's $t$-test).

of pheromone biosynthesis remaining in desat1 $R N A i$ flies.

For desatF RNAi flies, similar results have been described with another driver that targets expression in both oenocytes and fat body: the disappearance of dienes, which are replaced by monoenes of lower molecular weight [22]. This phenotype is due to the action of EloF, a female-specific elongase, which has been shown to elongate fatty acids to very long fatty acids (up to C30) [22]. This elongase is highly active in elongating dienic fatty acid precursors, and can also elongate monoenes, but with less efficiency. This would explain the particular profile of desatF RNAi females, very similar to that of males, with high levels of 7-T and 7-P, but with some other monoenes of a higher size, such as 7-heptacosene (C27:1). The main interest of the present study is to confirm that desatF expression is restricted to the oenocytes, and to compare the courtship of wild-type males toward both RNAi knocked-down females in the same conditions.

Overexpression of desat 1 in oenocytes led to about a $10 \%$ and $25 \%$ increase in pheromones in males and females, respectively. The use of three independent lines gave similar results. These figures are relatively small, compared to the $57 \%$ increase in female pheromones resulting from the overexpression of desatF [15]. This moderate effect of desat 1 overexpression could be due to a large expression of desat 1 in oenocytes, which is already sufficient for a whole synthesis of hydrocarbons. Approximately half of the desat1 transcripts occur in the oenocytes. This figure is high, even if it may be overestimated, due to a faint expression of 1407-GAL4 in tissues such as the gut, besides a large expression in oenocytes [5]. It must also be 
A

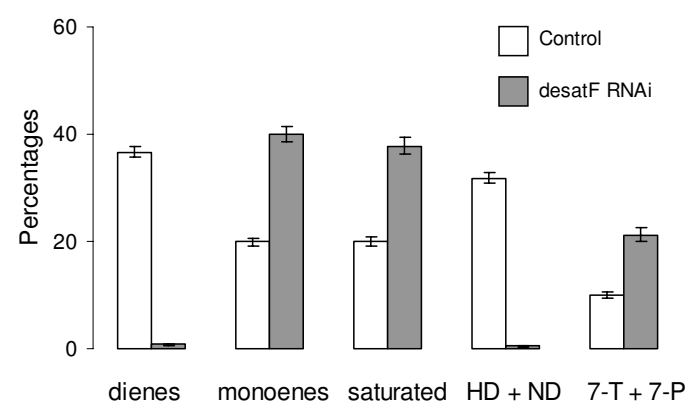

B

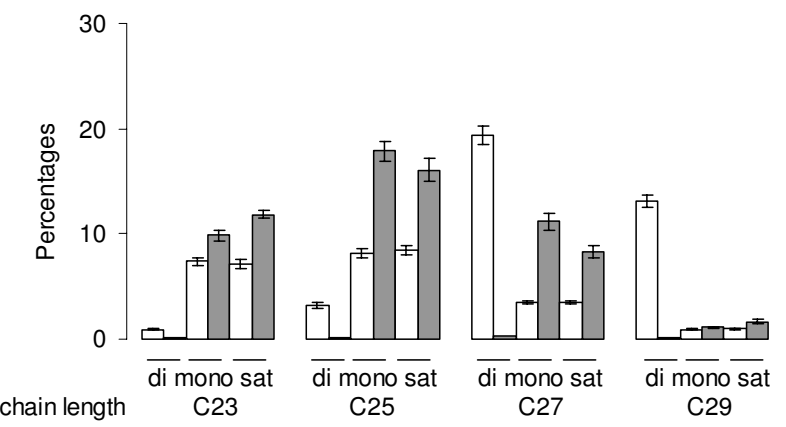

Figure 3

RNAi knock-down of desatF in oenocytes leads to a dramatic decrease in dienes counter-balanced by an increase in monoenes. A. Percentages of different classes of hydrocarbons (dienes, monoenes, saturated, female pheromones, male-type pheromones) B. Percentages of the specific hydrocarbons according to their class and their length. All the hydrocarbon differences between control (1407GAL4/+) and desatF knocked-down lines (I407-GAL4/UASdesatF RNAi) were significantly different $(P<0.001$, Student's $t$-test).

remembered that desat 1 is a pleiotropic gene, and its function in oenocytes is not limited to the synthesis of hydrocarbons, but also of other lipids, of fatty acids of the membranes, etc. On the other hand, desatF, which is specifically expressed in oenocytes, seems to be used only for pheromone production, as it is not expressed in species which have no dienic hydrocarbons [15]. The number of desatF transcripts is only $11 \%$ of the number of desat 1 transcripts in oenocytes, and might be the limiting factor to a full synthesis of dienes. In fact, all the studies on the control of Drosophila female pheromones have shown an action of hormones (ecdysone) or neurotransmitters (dopamine) on the second desaturation step [23-25]. desatF, unlike desat1, seems very sensitive to hormonal conditions and appears to be the gene in which the regulation of female pheromones is exerted.
Another desaturase gene, desat2, has been evidenced in some African strain females that produce 5,9-HD in place of 7,11-HD [10]. This gene is responsible for the unsaturation of fatty acid precursors in position 5 and resembles the desatF gene, in that it seems to be used only for the production of 5,9-HD pheromones. A recent quantification of desat 2 transcripts in these 5,9-HD females estimated a similar number of transcripts as for desatF (0.7 attomole/female) [11].

The role of unsaturated hydrocarbons on courtship behavior in $D$. melanogaster has been widely studied. The involvement of unsaturated hydrocarbons with $27 \pm 2$ carbons has been described $[1,8]$, and the threshold action of the 7,11-HD has been evaluated as near the nanogram level [26]. In a previous study with a desat1 mutant, the four-fold decrease in both 7-monoenes and 7,11-dienes in females was accompanied by a $50 \%$ increase in courtship latency [14]. In the present study the effect of desat1 knock-down is followed by a $78 \%$ decrease in 7 monoenes (similar to that for desat1 mutant), but also a $90 \%$ decrease in 7,11-dienes and a four-fold increase in courtship latency. The longer delay to courtship for desat 1 RNAi females, compared to the mutant ones, can thus be inferred from the lack of 7,11-dienes.

Knock-down of desatF gene in flies resulted in the disappearance of desatF transcripts, while only $2 \%$ of pheromones remained, and the levels of 7-P and 7-T were multiplied by two and three, respectively. These flies elicited the same courtship as wild-type females. Thus, monoenic hydrocarbons, such as 7-P and 7-H, can replace 7,11-dienes for courtship latency. However some parameters, such as copulation attempts and copulation latency, were much affected. In a previous study, similar results were obtained, using a more ubiquitous driver, with an effect only on copulation attempts and copulation latency [15]. These results tend to show that these two latter courtship parameters are affected by the presence of dienes.

desat 1 is a gene which is essential for the fly, contrary to desatF. When desatF is knocked-down, larger amounts of monoenes are synthesized. Some of these monoenes, especially 7-heptacosene, have been shown to play a role in courtship $[8,26]$. When desat 1 is knocked-down, only saturated hydrocarbons can be synthesized and none of these hydrocarbons have been shown to play a role in courtship. Actually, desat 1 has a larger influence on pheromones and courtship, but, in wild-type flies, its expression level does not seem to vary much (wild-type flies have similar amounts of unsaturated hydrocarbons); at the opposite, wild-type flies exhibit different amounts of pheromones (dienes) and desatF seems very sensitive to environmental and hormonal changes (C W-T, non published data). 


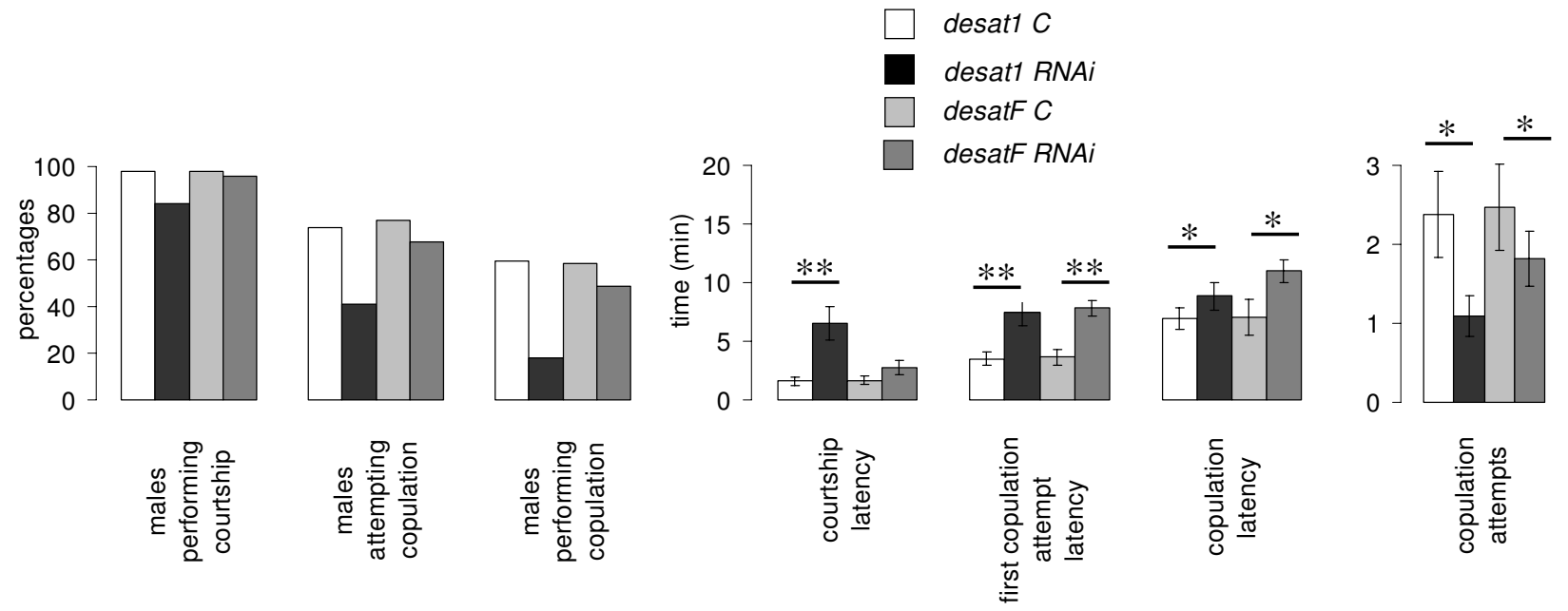

Figure 4

RNAi knock-down of desat I and desatF in females had very different effects on wild-type male courtship, with more marked effects in the former females. Mean courtship parameters $( \pm S E M)$ of 4-day-old tester Canton-S male flies with desat I RNAi females (controls in white, desat I RNAi in black) and desatF RNAi females (controls in light grey, desatF RNAi in grey). $\mathrm{n}>40$ for all tests. Means with $*$ and $* *$ were significantly different with the Mann-Whitney $U$-test $(P<0.05$ and 0.01 , respectively).

\section{Conclusion}

D. melanogaster and D. simulans diverged only 2.5 million years ago [27]. Both species differ by their cuticular hydrocarbons, the second species being sexually monomorphic, with large amounts of 7-T and 7-P in males and females [28]. Both species also share the same ecological niche [29]. The gene desatF is present in D. simulans genome, but not transcribed [15]. In a previous paper, the rapid evolution of desatF promoter has been evidenced and the importance of desatF in speciation suggested [30]. The present study shows that a knock-out of desatF in D. melanogaster has dramatic effects on 7,11-dienes, but that these effects may be partially counter-balanced by the large increases in 7-monoenes at the courtship-behavior level. One might suppose that evolution first inactivated desatF in D. simulans ancestors, permitting - almost - normal courtship behavior and reproduction, followed by other

Table I: About half of the total desat l transcripts are located in the oenocytes.

\begin{tabular}{llll}
\hline Genotype & transcripts & males & females \\
\hline I407-GAL4/+ & desatI & $0.62 \pm 0.11$ & $1.02 \pm 0.16$ \\
|407-GAL4/desatI RNAi & desatI & $0.32 \pm 0.08$ & $0.55 \pm 0.03$ \\
I407-GAL4/+ & desatF & 0 & $0.06 \pm 0.01$
\end{tabular}

The number of desat $\boldsymbol{F}$ transcripts represents only $10 \%$ of the number of desat I transcripts; all desatF transcripts are located in the female oenocytes.

The values represent the means of 4 determinations \pm SEM of samples from separate experiments and are given in attomoles/ $\mu \mathrm{g}$ total RNA. modifications at the reception level. These studies should bring about better understanding of how speciation has occurred in the Drosophila subgroup.

\section{Methods \\ Drosophila strains}

Flies were maintained at $25^{\circ} \mathrm{C}$ with $12: 12$ light-dark (LD) cycles, on standard yeast/cornmeal/agar medium. Virgin females were collected 0-6 hr after emergence, grouped in food vials and used 4 days later for hydrocarbon, fatty acid analyses and courtship study. Males were also collected 0$6 \mathrm{hr}$ after emergence and grouped in food vials for 4 days for hydrocarbon and fatty acid analyses. For courtship experiments, males from the Canton-S strain were isolated at emergence and kept individually in a food vial for 4 days.

One GAL4 line was used: 1407-GAL4 line targets expression in oenocytes and not in fat body [5].

The UAS-desat1RNAi line, referred to as desat1i, was provided by the Kyoto National Institute of Genetics http:// kyotofly.kit.jp/cgi-bin/stocks/data search.cgi. The UASdesatFRNA $i$ line, referred to as desatFi, was constructed and described previously [15].

\section{Construction of UAS-desat I lines}

The desat1 cDNA clone from Canton-S [7]. was cloned between the 5' EcoRI and 3' XbaI sites in $p\{U A S T\}$ plasmid [31]. Transgenic flies were generated by $P$-mediated germ- 
line transformation by Ann Mari Voie (EMBL) [32]. Multiple UAS-desat1i lines were obtained. Three lines, containing a single insert on the second chromosome, were used for genetic analysis.

\section{Crosses and data analysis}

The GAL4 females were crossed to UAS-desat1/Cy, UASdesat1i/Cy or UAS-desatFi/Cy males. The progeny was used for the experiments: the non $\mathrm{Cy}$ flies (expressing both GAL4 and the various UAS) were compared to the Cy control flies (expressing only GAL4). Data were analyzed using Student's $t$-test or one-way ANOVA.

\section{Hydrocarbon analysis}

Hydrocarbons were extracted from individual flies in heptane and analyzed by gas chromatography (Perichrom) as previously described [10]. They were quantified as percentages of total hydrocarbons. Data are presented as mean percentages \pm SEM of total hydrocarbons $(n=10$ for all tests). Only the hydrocarbons that show variations are represented.

\section{Analysis of courtship behavior}

One four-day-old virgin female (control or mutant) was tested with one four-day-old Canton-S male at $25^{\circ} \mathrm{C}$ for $20 \mathrm{~min}$, as previously described [33]. The following courtship parameters were measured: the percentage of males performing courtship (wing vibration), the percentage of males attempting copulation, the percentage of males successfully copulating, courtship latency (time from the introduction of the male into the female-containing observation chamber until wing vibration occurs), copulation attempts latency, copulation latency, ( $\mathrm{n}>40$ for all tests).

\section{Quantification of desat I and desatF transcripts}

The quantification of desaturase RNA was performed using the competitive quantitative polymerase chain reaction (PCR). This technique, which presents the advantage of high sensitivity, has been developed and has already been used for desat1 quantification [14]. In brief, a homologous internal DNA standard is used, differing only from desat $1 \mathrm{cDNA}$ by the presence of a short intron. The amplification of the two templates (desat1 cDNA together with the standard at different concentrations) permits desat 1 transcripts to be quantified quite easily.

The same technique was developed for desatF. For the construction of the competitor, we used the two restriction sites EcoRI and BamHI, naturally occurring in the sequence and $465 \mathrm{pb}$ distant from each other. desatF ORF (1068 bp) was cloned in a SK Bluescript, using XbaI and HindIII restriction sites of the plasmid. The clone was then digested with EcoRI and BamHI, liberating a 465 bp frag- ment and relegated, yielding to a 603 bp truncated orf, which was used as a competitor.

\section{Authors' contributions}

CW-T did the pheromone analyses and wrote the paper; IG performed the desat 1 and desatF quantification and participated to the writing; YFK performed the courtship behaviour and participated to the writing.

\section{Acknowledgements}

Funding was provided by the French Ministry of Research and Education (for I G and Y F K) and by the Centre National de la Recherche Scientifique (for C W-T).

\section{References}

I. Antony C, Jallon J-M: The chemical basis for sex recognition in Drosophila melanogaster. J Insect Physiol I 982, 28:873-880.

2. Jallon JM: A few chemical words exchanged by Drosophila during courtship and mating. Behav Genet 1984, I 4(5):44 I-478.

3. Greenspan RJ, Ferveur JF: Courtship in Drosophila. Annu Rev Genet 2000, 34:205-232.

4. Ejima A, Griffith LC: Courtship initiation is stimulated by acoustic signals in Drosophila melanogaster. PLOS ONE 2008, 3(9):e3246.

5. Ferveur JF, Savarit F, O'Kane CJ, Sureau G, Greenspan RJ, Jallon JM: Genetic feminization of pheromones and its behavioral consequences in Drosophila males. Science 1997, 276(53 | 8): I 555-I558.

6. Blomquist G, Dillwith JW, Adams TS: Biosynthesis and endocrine regulation of sex pheromone production in Diptera. In Pheromone Biochemistry Edited by: Prestwitch GD, Blomquist G. London: Academic Press; 1987:217-250.

7. Pennanec'h M, Ferveur J-F, Pho DB, Jallon J-M: Insect fatty acid related pheromones: a review of their biosynthesis, hormonal regulation and genetic control. Ann Soc Ent Fr 1991, 27:245-263.

8. Antony C, Davis TL, Carlson DA, Péchiné J-M, Jallon J-M: Compared behavioral responses of male Drosophila melanogaster (Canton S) to natural and synthetic aphrodisiacs. J Chem Ecol I985, I I(I2): I6I7-1629.

9. Wicker-Thomas C, Henriet C, Dallerac R: Partial characterization of a fatty acid desaturase gene in Drosophila melanogaster. Insect Biochem Mol Biol 1997, 27(I I):963-972.

10. Dallerac R, Labeur C, Jallon JM, Knipple DC, Roelofs WL, WickerThomas C: A delta 9 desaturase gene with a different substrate specificity is responsible for the cuticular diene hydrocarbon polymorphism in Drosophila melanogaster. Proc Nat Acad Sci USA 2000, 97( I 7):9449-9454.

II. Wicker-Thomas C, Chertemps T: Chapter 4: Molecular biology and genetics of hydrocarbon production. In Insect hydrocarbons: Biology, Biochemistry and Chemical Ecology Edited by: Blomquist GA, Bagnères AG. Cambridge: Cambridge University Press; 2010 in press.

12. Labeur C, Dallerac R, Wicker-Thomas C: Involvement of desat I gene in the control of Drosophila melanogaster pheromone biosynthesis. Genetica 2002, I |4(3):269-274.

13. Marcillac F, Bousquet F, Alabouvette J, Savarit F, Ferveur JF: A mutation with major effects on Drosophila melanogaster sex pheromones. Genetics 2005, I71(4):1617-1628.

14. Ueyama M, Chertemps T, Labeur C, Wicker-Thomas C: Mutations in the desat I gene reduces the production of courtship stimulatory pheromones through a marked effect on fatty acids in Drosophila melanogaster. Insect Biochem Mol Biol 2005, 35(8):911-920.

15. Chertemps T, Duportets L, Labeur C, Ueyama M, Wicker-Thomas C: A female-specific desaturase gene responsible for diene hydrocarbon biosynthesis and courtship behaviour in Drosophila melanogaster. Insect Mol Biol 2006, I 5(4):465-473.

16. Pho DB, Pennanec'h M, Jallon J-M: Purification of adult Drosophila melanogaster lipophorin and its role in hydrocarbon transport. Archives of insect biochemistry and physiology 1996, 3 I (3):289-303. 
17. Dantuma NP, Pijnenburg MA, Diederen JH, Horst DJ Van der: Multiple interactions between insect lipoproteins and fat body cells: extracellular trapping and endocytic trafficking. J Lipid Res 1998, 39(9): |877-|888.

18. Schal C, Sevala V, Capurro ML, Snyder TE, Blomquist GJ, Bagneres AG: Tissue distribution and lipophorin transport of hydrocarbons and sex pheromones in the house fly, Musca domestica. J Insect Sci 200 I, I ( I 2): I - I I.

19. Fan Y, Zurek L, Dykstra MJ, Schal C: Hydrocarbon synthesis by enzymatically dissociated oenocytes of the abdominal integument of the German cockroach, Blattella germanica. Naturwissenschaften 2003, 90(3):121-126.

20. Fan Y, Eliyahu D, Schal C: Cuticular hydrocarbons as maternal provisions in embryos and nymphs of the cockroach Blattella germanica. J Exp Biol 2008, 2 I I (Pt 4):548-554.

21. Chan Yong T, Jallon J-M: Synthèse de novo d'hydrocarbures potentiellement aphrodisiaques chez les Drosophiles. $C r$; hebd Séanc Acad Sci 1986, 303:197-202.

22. Chertemps T, Duportets L, Labeur C, Ueda R, Takahashi K, Saigo K, Wicker-Thomas C: A female-biased expressed elongase involved in long-chain hydrocarbon biosynthesis and courtship behavior in Drosophila melanogaster. Proc Natl Acad Sci USA 2007, I 04(I I):4273-4278.

23. Wicker C, Jallon J-M: Influence of ovary and ecdysteroids on pheromone biosynthesis in Drosophila melanogaster (Diptera: Drosophilidae). Eur J Entomol 1995, 92:197-202.

24. Wicker C, Jallon J-M: Hormonal control of sex pheromone biosynthesis in Drosophila melanogaster. J Insect Physiol 1995 41:65-70.

25. Marican C, Duportets L, Birman S, Jallon JM: Female-specific regulation of cuticular hydrocarbon biosynthesis by dopamine in Drosophila melanogaster. Insect Biochem Mol Biol 2004, 34(8):823-830.

26. Ferveur J-F, Sureau G: Simultaneous influence on male courtship of stimulatory and inhibitory pheromones produced by live sex mosaic Drosophila melanogaster. Proc $R$ Soc 1996, B263:967-973.

27. Hey J, Kliman RM: Population genetics and phylogenetics of DNA sequence variation at multiple loci within the Drosophila melanogaster species complex. Mol Biol Evol 1993, 10(4):804-822.

28. Jallon J-M, David J: Variations in cuticular hydrocarbons among the eight species of the Drosophila melanogaster subgroup. Evolution 1987, 4:294-302.

29. David J Tsacas J: Cosmopolitan, subcosmopolitan and widespread species: different strategies within the Drosophila family. CRSoc Biogeog 1981, 57:11-26.

30. Legendre A, Miao XX, Da Lage JL, Wicker-Thomas C: Evolution of a desaturase involved in female pheromonal cuticular hydrocarbon biosynthesis and courtship behavior in Drosophila. Insect Biochem Mol Biol 2008, 38(2):244-255.

31. Brand AH, Perrimon N: Targeted gene expression as a means of altering cell fates and generating dominant phenotypes. Development 1993, I I 8(2):40|-4I5.

32. Rubin GM, Spradling AC: Genetic transformation of Drosophila with transposable element vectors. Science 1982, 218(4570):348-353.

33. Jallon JM, Hotta $Y$ : Genetic and behavioral studies of female sex appeal in Drosophila. Behav Genet 1979, 9(4):257-275.
Publish with Biomed Central and every scientist can read your work free of charge

"BioMed Central will be the most significant development for disseminating the results of biomedical research in our lifetime. "

Sir Paul Nurse, Cancer Research UK

Your research papers will be:

- available free of charge to the entire biomedical community

- peer reviewed and published immediately upon acceptance

- cited in PubMed and archived on PubMed Central

- yours - you keep the copyright
BioMedcentral 